\title{
Diagnostic accuracy of carotid intima media thickness by B-mode ultrasonography in coronary artery disease patients
}

Nasir Raza Zaidi, Syed Amir Gilani, Riffat Mehboob, Humaira Waseem, Amber Hassan

University Institute of Radiological Sciences and Medical Imaging Technology, University of Lahore, Lahore, Pakistan

Submitted: 12 October 2019

Accepted: 10 April 2020

Arch Med Sci Atheroscler Dis 2020; 5: e79-e84

DOI: https://doi.org/10.5114/amsad.2020.95651

Copyright $\odot 2020$ Termedia \& Banach

\section{Abstract}

Introduction: Cardiovascular diseases (CVD) are the main cause of premature deaths worldwide, and atherosclerosis (AS) is a major risk factor associated with them. B-mode ultrasound is a well-validated research tool that has been translated increasingly into clinical practice. The aim of the study was to assess the diagnostic accuracy of carotid intima media thickness by B-mode ultrasonography in coronary artery disease patients.

Material and methods: This was a case control study, including 100 cases and the same number of controls. Patients with positive angiographic findings and chest pain were considered as cases and those without as negative. Duplex carotid ultrasound was used to detect intima-media thickness (IMT). B-mode real-time ultrasonic images were obtained with a $7 \mathrm{MHz}$ transducer. An intima media thickness of $0.6 \mathrm{~mm}$ was considered as being without plaque.

Results: The angiographic findings were single-vessel disease, double-vessel disease, and triple-vessel disease in $18 \%, 11.5 \%$, and $20.5 \%$ of cases, respectively, while there were no findings in controls. There was plaque formation in $14.5 \%$ and calcification in $12 \%$ of the cases. Sensitivity of B-mode ultrasonography was found to be $78 \%$, specificity $75 \%$, positive predictive value $75.72 \%$, and negative predictive value $77.31 \%$.

Conclusions: Carotid ultrasonography can be utilised as a valuable screening tool due to having several advantages, including ease of application, reproducibility, low cost, and strong correlation with atherosclerosis.

Key words: coronary artery disease, cardiovascular disease, cerebrovascular disease, intima media thickness, B-mode ultrasound.

\section{Introduction}

Cardiovascular diseases (CVDs) are on the rise on the global scale, causing premature death and rendering a huge socioeconomic burden. Their underlying pathogenesis is mainly atherosclerosis (AS). CVDs may progress to develop coronary artery disease (CAD), cerebrovascular disease, peripheral vascular disease, and coronary heart disease (CHD) and hence may lead to myocardial infarction, cardiac arrhythmias, cardiac arrest, or stroke [1]. CHD alone is responsible for one third of all mortalities in the young adult population in developed countries [2, 3].

Age, sex, and familial predisposition [4] are also irreversible accompaniments to our lives, but others are clearly or potentially reversible, like

\author{
Corresponding author: \\ Prof. Riffat Mehboob \\ Faculty of Allied Health \\ Sciences \\ The University of Lahore \\ Lahore, Pakistan \\ E-mail: mehboob.riffat@ \\ gmail.com
}


hypertension, hyperlipidaemia, cigarette smoking, and diabetes mellitus. Age has a dominant influence on the development of clinically significant AS [1,5], although some death rates from CHD are significantly higher in males until age $75-85$ years, when the incidences in males and females approach equality. Significantly, myocardial infarction is uncommon in premenopausal women and in the age group of 35 to 55 years. The mortality rate of white males is over five times that of white females. Naturally, other risk factors influence the relative risk of CHD in females; the rate is increased in women with a history of heavy smoking, hypertension, or diabetes.

Despite advancement of atherosclerosis, symptoms are not expressed in patients with CHD. However, it has a poor prognosis compared to patients without CHD. Hence, it is of utmost importance to identify at-risk, asymptomatic patients, in order to prevent mortality and morbidity through medical interventions [6]. Sonographic measurement of arteries and their imaging has been suggested as a diagnostic tool for CVD [7].

Carotid intima-media thickness (CIMT) and carotid plaque burden are considered as predictors of cardiovascular risk [8]. In 2000, an American Heart Association (AHA) conference concluded that CIMT "can now be considered for further clarification of CHD risk assessment at the request of a physician", provided that it is performed by an experienced laboratory [9]. In a very recent study conducted by Bulut et al., CIMT was also reported to determine the target organ damage in patients with CAD [10]. CIMT was significantly linked to CVDs including myocardial infarction, transient ischaemic attack, etc. [11]. CIMT measurement with B-mode ultrasound is a sensitive technique to estimate the AS plaques and risk of developing CVD [12].

Morphological arterial wall abnormalities by B-mode imaging were previously done for the evaluation of fibromuscular dysplasia and were considered non-invasive and accurate [13]. Recently, it has also been used in a computer-aided multimodal diagnostic technique for prostate cancer and was found to be helpful [14]. It also has diagnostic applications in many other diseases of the abdomen, kidney, breast, liver, and thyroid, as well as musculoskeletal and gynaecological diseases, etc.) [15].

In Pakistan, many hospitals do not have the facility of angiography; therefore, a screening test "B-mode ultrasonography of carotid artery" is used worldwide, and this is why this test is being compared with a confirmatory/diagnostic test/ gold standard test (angiography). The results in different countries have revealed good reliability, sensitivity, specificity, predictive value, accuracy, and precision [16]. Carotid plaques are a progressive stage of AS and a risk factor for CVD, and different imaging methodologies are being used these days for its diagnosis. For this purpose, the thickness of the carotid artery wall is measured, which is an indicator of the initial phase of AS [11]. This study was conducted to assess the CIMT measurements in carotid artery disease patients by B-mode ultrasonography and its diagnostic accuracy.

\section{Material and methods}

A case control study was conducted at the Radiology Department of Mayo Hospital Lahore. A total of 200 patients were selected through convenient sampling technique, from whom 100 were cases and another 100 were controls. All the patients presented with chest pain. Angiography test was performed to have a clear picture of open, narrow, and blocked and narrow arteries and veins. Patients with positive angiography test were subjected to carotid ultrasonography and considered as cases. The other 100 patients of chest pain with normal angiography test were considered as controls after obtaining their consent. Patients on any medication, unable to hold their head still, or with high bifurcation of the carotid artery were excluded. The mean age of cases was $51.62 \pm 11.23$ years and of controls was 47.15 +9.35. In cases, there were 77 males and $23 \mathrm{fe}$ males, and in controls 52 males and 48 females. The study was approved by the Ethical Review Board of the University of Lahore, Pakistan.

Both the sonographer and patient were positioned properly to facilitate high-quality, reproducible images. The sonographer was positioned at the head of the patient, with enough space to rest an elbow on the patient's head. The patient was positioned supine on the scan bed with their head resting comfortably [17]. Duplex carotid ultrasound was used to detect intima-media thickness (IMT). B-mode real time ultrasonic images with a $7 \mathrm{MHz}$ transducer were used. Most patients could be scanned at a standard depth of $4 \mathrm{~cm}$. The resolution decreases with increasing imaging depth. The typical pixel size when imaging at a $4 \mathrm{~cm}$ depth is approximately $0.11 \mathrm{~mm}$.

An ultrasound machine with a linear array transducer working at a minimum frequency of $7 \mathrm{MHz}$ and depth of $4 \mathrm{~cm}$ was used for the measurement of CIMT. Zooming of the images is not recommended because these measurements are very small, and even a difference of one digital pixel matters a lot [16]. Carotid ultrasound imaging should follow a scanning protocol from a large epidemiologic study that reported CIMT values in percentiles by age, sex, and race/ethnicity [18]. The data acquired included transverse and lon- 
gitudinal images of common carotid parameters throughout visualised vessels. IMT up to $0.6 \mathrm{~mm}$ (mean of one side) was considered as the absence of any plaque [19]. Fifty percent or greater stenosis of the studied vessel was considered haemodynamically significant. A self-structured questionnaire was used to collect the data. Mean \pm SD was used to describe the quantitative variables. The $\chi^{2}$ test was used to see the association among the different variables. Data were analysed with SPSS Version 21.0.

The CIMT portion of the recommended scanning protocol is based on the Atherosclerosis Risk in Communities (ARIC) study protocol because it was a large study with published nomograms for CIMT values in the age range that is usually most appropriate for screening. Furthermore, in the ARIC study, both increasing CIMT and carotid plaque presence independently predicted CVD events, and the scanning methods are reproducible in most clinical laboratories $[18,20]$. The region to be measured includes the far wall of the distal $1 \mathrm{~cm}$ of the CCA. The distal common carotid artery (CCA) should be perfectly horizontal on the screen with simultaneous double lines in the near and far walls of the CCA. This is accomplished by a combination of small adjustments in transducer tilt, rotation, and differential pressure of the proximal-to-distal end of the probe (heeltoe movement). After the optimal angle of incidence (OAI) is identified, the distal $1 \mathrm{~cm}$ of the CCA should be imaged from two additional complimentary angles, approximately $45^{\circ}$ anteriorly and posteriorly to cover a representative range of the neck circumference (anterior, lateral, and posterior). If the patient's OAI is extremely anterior or posterior, two additional images approximately $45^{\circ}$ apart should be obtained. Applying different degrees of pressure and use of gel as an acoustic standoff will improve resolution and reduce artefacts.

When evaluating for the presence or absence of plaque in conjunction with measuring CCA, CIMT offers a better representation of sub-clinical vascular disease and CVD risk than only measuring CIMT. Carotid plaque is defined as the presence of focal wall thickening that is at least $50 \%$ greater than that of the surrounding vessel wall or as a focal region with CIMT greater than $1.5 \mathrm{~mm}$, which protrudes into the lumen, and which is distinct from the adjacent boundary [21].

Measurement of CIMT involves tracing the blood-intima and media-adventitia interfaces of the far wall using a leading edge-to-leading edge technique. The best image for CIMT measurement demonstrates the blood-intima and media-adventitia boundaries clearly. The reader should be able to see these interfaces on both near and far walls of the carotid artery to ensure that the so- nographer has imaged the vessel through its truest diameter, otherwise the CIMT may be thicker or thinner than is anatomically correct [22]. If the images do not show a complete $1 \mathrm{~cm}$ segment, the tracing maybe shortened. Tracing of interfaces that are not clearly visualised should be avoided. If plaques are detected in the segment being measured, they should be traced as part of the CIMT, because they appear to have been included in CIMT measurements in most of the epidemiologic studies in an alternate reading protocol, based on published nomograms and risk prediction associations, which also may be used. In general, segments should be measured in triplicate and CIMT values averaged. Most studies provided reference values in used manual reading techniques; however, semiautomated border detection programs were used by some. Semiautomated border detection programs are widely available and, when used on high-quality images, tend to improve reproducibility and shorten reading time, especially among newer readers [23].

Mean CIMT values from the far walls of the right and left CCAs (mean-mean) should be reported. Use of additional segments or maximum values is an alternative if there is local expertise and these measurements can be mapped to normative values with published associations to CVD risk. Most reading software will report meanmean (average of segmental mean CIMT values) and mean-maximum (average of segmental maximum CIMT values) CIMT values. Mean-mean values are more reproducible because multiple points along the traced segment are averaged, but they are less sensitive to change. Mean maximum values are more sensitive to change, but are less reproducible, because they are derived from a single point (or regional maximum) measurement along the $1 \mathrm{~cm}$ region.

\section{Results}

In coronary artery disease patients, 36 patients had single-vessel, 23 had double-vessel, and 41 patients had triple-vessel diseases. All 100 control patients had normal angiographic findings. There is a statistically significant association between angiographic findings and coronary artery disease. The proportion of plaque formation among coronary artery disease was greater (29\%) than in their respective controls $(0 \%)(p<0.05)$. The proportion of calcification among coronary artery disease was greater $(26 \%)$ than in their respective controls $(0 \%)(p<0.05)$ (Table I).

Among the cases 36 patients had single-vessel disease, 23 patients had double-vessel disease, and 41 patients had triple-vessel disease. The mean \pm SD of IMT thickness on right side in singlevessel disease patients was $0.74 \pm 0.24$, in dou- 
Table I. Angiographic findings in cases and controls

\begin{tabular}{|lcccc|}
\hline Angiography findings & Cases & Control & Total & $P$-value \\
\hline Single vessel disease & 36 & 0 & 36 & $<0.001$ \\
\hline Double vessel disease & 23 & 0 & 23 & \\
\hline Triple vessel disease & 41 & 0 & 41 & \\
\hline Normal coronary vessels & 0 & 100 & 100 & $<0.001$ \\
\hline Total & 100 & 100 & 200 & \\
\hline \begin{tabular}{l} 
Plaque: \\
\hline Yes
\end{tabular} & 29 & 0 & 29 & \\
\hline No & 71 & 100 & 200 & $<0.001$ \\
\hline Total & 100 & 100 & 24 \\
\hline Calcification: & 24 & 100 & 176 \\
\hline Yes & 76 & 100 & 200 \\
\hline No & 100 & 0 & \\
\hline Total & & 100 & \\
\hline
\end{tabular}

ble-vessel disease patients it was $0.94 \pm 0.49$, and in triple-vessel disease patients it was $0.90 \pm 0.31$. The mean \pm SD of IMT thickness on the left side in single-vessel disease patients was $0.76 \pm 0.32$, in double-vessel disease patients it was $0.92 \pm 0.46$, and in triple-vessel disease patients the thickness was $1.02 \pm 0.51$ (Table II). Diagnostic accuracy of angiography and CIMT B-Mode ultrasonography showed $78 \%$ sensitivity, $75 \%$ specificity, $75.72 \%$ positive predictive value, and $77.31 \%$ negative predictive value (Table III).

\section{Discussion}

Cardiovascular diseases are the main and silent cause of death in the western world [24] because they remain mostly undiagnosed unless a sudden episode occurs and symptoms appear. Hence, the importance of establishing accurate diagnostic

Table II. Mean and standard deviation of intima media in patients of coronary artery disease with respect to angiographic findings

\begin{tabular}{|cccccc|}
\hline \multicolumn{4}{|c}{ IMT thickness - right side $[\mathrm{mm}]$} & \multicolumn{3}{c|}{ IMT thickness - left side [mm] } \\
\hline SVD $(n=36)$ & DVD $(n=23)$ & TVD $(n=41)$ & SVD $(n=36)$ & DVD $(n=23)$ & TVD $(n=41)$ \\
\hline $0.74 \pm 0.24$ & $0.94 \pm 0.49$ & $0.90 \pm 0.31$ & $0.76 \pm 0.32$ & $0.92 \pm 0.46$ & $1.02 \pm 0.51$ \\
\hline
\end{tabular}

SVD - single-vessel disease, DVD - double-vessel disease, TVD - triple-vessel disease.

Table III. Testing a case of carotid artery disease (B-mode ultrasonography) versus confirmatory test (angiography) of coronary artery disease

\begin{tabular}{|c|c|c|c|c|}
\hline & \multirow[t]{2}{*}{ Test result } & \multicolumn{3}{|c|}{ Angiography } \\
\hline & & Positive & Negative & Total \\
\hline \multirow{2}{*}{$\begin{array}{l}\text { CIMT B-mode } \\
\text { ultrasonography }\end{array}$} & Positive & 78 & 11 & 89 \\
\hline & Negative & 22 & 89 & 111 \\
\hline Total & & 100 & 100 & 200 \\
\hline Sensitivity & \multicolumn{4}{|c|}{$78 \%$} \\
\hline Specificity & \multicolumn{4}{|c|}{$75 \%$} \\
\hline Positive predictive value & \multicolumn{4}{|c|}{$75.72 \%$} \\
\hline Negative predictive value & \multicolumn{4}{|c|}{$77.31 \%$} \\
\hline
\end{tabular}


tools for early diagnosis and preventive measures becomes evident. CIMT is a predictive, non-invasive, established, and economical marker for the assessment of at-risk individuals before the actual onset of disease symptoms.

CIMT measurements by ultrasound may be used for the assessment of AS and risk to develop CAD. Current findings indicate $78 \%$ sensitivity and $75 \%$ specificity, which shows that it is able to correctly diagnose the disease with accuracy. A previous study conducted by ARIC also reported an association of increased thickness of carotid artery with increased risk of CAD by B-mode ultrasonography [25].

Coskun et al. studied 100 patients, of whom 39 were without a non-critical coronary lesions, and the remaining 61 patients had at least one lesion more than $50 \%$ within the main branches of the coronary arteries. Among patients whose angiographic findings were positive, 77 were males and 23 were females [26]. Another study done by Haberka et al. suggested that carotid vascular indices such as CIMT, carotid extra media thickness (CEMT), combined PATIMA index, and carotid artery stenosis may serve as predictors and indicators of CAD. They enrolled 322 patients who were scheduled for coronary angiography. They had equal rates of single, double, and triple vessel disease. A total of 158 patients were diagnosed with CAD [27]. A cohort study was conducted in Denmark on coronary angiographies patients during 2003-2012. 78,195 patients were enrolled, among whom 32,061 (41\%) patients had zero-vessel disease, 6205 (7.93\%) had diffused-vessel disease, 20202 (25.83\%) had single-vessel disease, 10,675 (13.65\%) had double-vessel disease, and 9038 (11.55\%) had triple-vessel disease [28]. In our study, $36 \%$ patients had single-vessel, $23 \%$ had double-vessel, and $41 \%$ had triple-vessel disease.

CIMT measurement is a non-invasive technique to predict CAD to some extent, but its precision increases if coupled with inflammatory markers such as high-sensitivity C-reactive protein (hsCRP) [29]. Thus, CIMT may be used as a predictor of AS [30]. In some other studies CIMT was also found to be associated with CADs [31]. However, these studies are done in the western world most of the time. No study is available for a South Asian population. The current study findings also confirmed the same facts in a Pakistani population.

Polak and O'Leary also suggested that CIMT may add to the Framingham risk score (FRS) for the prediction of CAD. They measured CIMT in 2965 participants, and CAD disease outcomes were monitored for the subsequent 7.2 years. Reclassification of CAD was done on the basis of 8-year FRS category. A total of 296 participants had CAD. It was concluded that the maximum internal and mean common CIMT can both diag- nose CAD, but the maximum CIMT enhances the FRS of CAD [32]. The presence of plaque was significantly associated with plaque formation in the CAD patients. Results from another study showed that the carotid AS was significantly related to the presence of triple-vessel disease. Furthermore, it was reported by Polak and O'Leary that CIMT is significantly correlated with the extent of CAD [32]. In the present study, plaque formation and calcification were observed in $29 \%$ and $24 \%$ of the cases, respectively.

Dynamic range (DR) setting has also been reported to have an impact on CIMT measurements, especially the lower DRs [19]. It has also been suggested that the DR be assessed in order to increase the reliability and validity of measurements of CIMT. Strict ultrasound protocols are highly recommended, to emphasise the reproducibility of the results. A population including larger data sets should be assessed in further studies to standardise the normal ranges within a particular population. Because it is one of the pioneer studies from South East Asia, to the knowledge of the researchers, it may be considered as a pilot study and may provide a foundation for further studies. Diagnostic accuracy of B-mode ultrasound in different populations may be very helpful in setting up standardised protocols globally. Future studies on risk factor assessment for CAD and its prevention may also be conducted.

In conclusion, CIMT has been observed to be reliable predictor and biomarker for the diagnosis and prognosis of CAD. Carotid plaques may provide a deep insight into the pathological mechanisms and extent of disease. B-mode ultrasonography is a precise, easy, economical technique for the diagnosis of CAD. Hence, it should be done as routine for the risk assessment of CAD cases. In Pakistan many hospitals do not have the facility of angiography; therefore, such a screening test for CIMT could be used against a gold standard test.

\section{Conflict of interest}

The authors declare no conflict of interest.

\section{References}

1. Flora GD, Nayak MK. A brief review of cardiovascular diseases, associated risk factors and current treatment regimes. Curr Pharm Des 2019; 25: 4063-84.

2. Sanchis-Gomar F, Perez-Quilis C, Leischik R, Lucia A. Epidemiology of coronary heart disease and acute coronary syndrome. Ann Transl Med 2016; 4: 256.

3. Mozaffarian D, Benjamin EJ, Go AS, et al. Heart disease and stroke statistics-2016 Update: a report from the American Heart Association. Circulation 2016; 133: e38-360.

4. Zheng Q, Jiang J, Huo Y, Chen D. Genetic predisposition to type 2 diabetes is associated with severity of coronary artery disease in patients with acute coronary syndromes. Cardiovasc Diabetol 2019; 18: 131. 
5. Insull W Jr. The pathology of atherosclerosis: plaque development and plaque responses to medical treatment. Am J Med 2009; 122(1 Suppl): S3-14.

6. Maddox TM, Stanislawski MA, Grunwald GK, et al. Nonobstructive coronary artery disease and risk of myocardial infarction. JAMA 2014; 312: 1754-63.

7. Taylor AJ, Merz CN, Udelson JE. 34 ${ }^{\text {th }}$ Bethesda Conference: executive summary - can atherosclerosis imaging techniques improve the detection of patients at risk for ischemic heart disease? J Am Coll Cardiol 2003; 41: 1860-2.

8. Paraskevas $\mathrm{KI}$, Sillesen $\mathrm{HH}$, Rundek $\mathrm{T}$, et al. Carotid intima-media thickness versus carotid plaque burden for predicting cardiovascular risk. Angiology 2020; 71: 108-11.

9. Greenland P, Abrams J, Aurigemma GP, et al. Prevention Conference V: Beyond secondary prevention: identifying the high-risk patient for primary prevention: noninvasive tests of atherosclerotic burden: Writing Group III. Circulation 2000; 101: E16-22.

10. Bulut A, Acele A, Donmez Y, et al. Aortic intima-media thickness can be used to determine target organ damage in adult patients with coronary artery disease risk factors. Arch Med Sci Atheroscler Dis 2019; 4: e183-90.

11. Nezu T, Hosomi N, Aoki S, et al. Carotid intima-media thickness for atherosclerosis. J Atheroscler Thromb 2016; 23: 18-31.

12. Amato M, Montorsi P, Ravani A, et al. Carotid intima-media thickness by B-mode ultrasound as surrogate of coronary atherosclerosis: correlation with quantitative coronary angiography and coronary intravascular ultrasound findings. Eur Heart J 2007; 28: 2094-101.

13. Cutler JJ, Campo N, Koch S. B-flow and B-mode ultrasound imaging in carotid fibromuscular dysplasia. J Neuroimaging 2018; 28: 269-72.

14. Zhang Q, Xiong J, Cai Y, Shi J, Xu S, Zhang B. Multimoda feature learning and fusion on B-mode ultrasonography and sonoelastography using point-wise gated deep networks for prostate cancer diagnosis. Biomed Tech (Berl) 2020; 65: 87-98.

15. Meiburger KM, Acharya UR, Molinari F. Automated localization and segmentation techniques for B-mode ultrasound images: a review. Comput Biol Med 2018 92: 210-35.

16. Naik V, Gamad RS, Bansod PP. Carotid artery segmentation in ultrasound images and measurement of intima-media thickness. Biomed Res Int 2013; 2013: 801962.

17. Mitchell CK, Aeschlimann SE, Korcarz CE. Carotid intimamedia thickness testing: technical considerations. Am Soc Echocardiogr 2004; 17: 690-2.

18. Howard G, Sharrett AR, Heiss G, et al. Carotid artery intimal-medial thickness distribution in general populations as evaluated by B-mode ultrasound. ARIC Investigators. Stroke 1993; 24: 1297-304.

19. Gaarder M, Seierstad T. Measurements of carotid intima media thickness in non-invasive high-frequency ultrasound images: the effect of dynamic range setting. Cardiovasc Ultrasound 2015; 13: 5.

20. Tzou WS, Douglas PS, Srinivasan SR, et al. Distribution and predictors of carotid intima-media thickness in young adults. Prev Cardiol 2007; 10: 181-9.

21. Touboul PJ, Hennerici MG, Meairs S, et al. Mannheim carotid intima-media thickness consensus (2004-2006) An update on behalf of the Advisory Board of the $3^{\text {rd }}$ and $4^{\text {th }}$ Watching the Risk Symposium, $13^{\text {th }}$ and $15^{\text {th }}$ European Stroke Conferences, Mannheim, Germany, 2004, and Brussels, Belgium, 2006. Cerebrovasc Dis 2007; 23: 75-80
22. Bots ML, Evans GW, Riley WA, et al. Carotid intima-media thickness measurements in intervention studies: design options, progression rates, and sample size considerations: a point of view. Stroke 2003; 34: 2985-94.

23. Gepner AD, Korcarz CE, Aeschlimann SE, et al. Validation of a carotid intima-media thickness border detection program for use in an office setting. J Am Soc Echocardiogr 2006; 19: 223-8

24. Van Camp G. Cardiovascular disease prevention. Acta Clin Belg 2014; 69: 407-11.

25. O'Leary DH, Polak JF, Kronmal RA, et al. Carotid-artery intima and media thickness as a risk factor for myocardial infarction and stroke in older adults. Cardiovascular Health Study Collaborative Research Group. N Engl J Med 1999; 340: 14-22.

26. Coskun U, Yildiz A, Esen OB, et al. Relationship between carotid intima-media thickness and coronary angiographic findings: a prospective study. Cardiovasc Ultrasound 2009; 7: 59.

27. Haberka $M$, Batys $M$, Matla $M$, et al. Carotid artery stenosis and ultrasound vascular indexes predict the coronary revascularization in patients with high cardiovascular risk scheduled for coronary angiography. Kardiol Pol 2019; 77: 1028-33.

28. Olesen KKW, Madsen M, Lip GYH, et al. Coronary artery disease and risk of adverse cardiac events and stroke. Eur J Clin Invest 2017; 47: 819-28.

29. Abhashi SA, Kryeziu FU, Nazreku FD. Increased carotid intima-media thickness associated with high hs-CRP levels is a predictor of unstable coronary artery disease. Cardiovasc J Afr 2013; 24: 270-3.

30. Centurion OA. Carotid intima-media thickness as a cardiovascular risk factor and imaging pathway of atherosclerosis. Crit Pathw Cardiol 2016; 15: 152-60.

31. Balta S, Aparci M, Ozturk C, et al. Carotid intima media thickness can predict coronary artery disease. Int J Cardiol 2015; 201: 331

32. Polak JF, O'Leary DH. Carotid intima-media thickness as surrogate for and predictor of CVD. Glob Heart 2016 11: 295-312 e3. 